\title{
Satellite Gateway Diversity in SDN/NFV-enabled satellite ground segment systems
}

\author{
T. Ahmed ${ }^{1}$, R. Ferrus ${ }^{2}$, R. Fedrizzi ${ }^{3}$, O. Sallent ${ }^{2}$, Nicolas Kuhn $^{4}$, Emmanuel Dubois ${ }^{4}$, Patrick Gelard ${ }^{4}$ \\ 1) CNRS-LaBRI (UMR5800), University of Bordeaux / Bordeaux INP, France \\ 2) Universitat Politècnica de Catalunya, Spain \\ 3) Center for Research and Telecommunication Experimentation for Networked communities, Italy \\ ${ }^{4)}$ Centre National d'Etudes Spatiales, France
}

\begin{abstract}
This paper explores how to use Software Defined Networking (SDN) and Network Functions Virtualization (NFV) as two emerging technologies to support Satellite Gateway Diversity (GWD) solution in the forward link to provide next generation satellite system with higher capacity enhancement, failover and resiliency management. In this context, the gateway handover along with efficient traffic steering techniques are used to cope with the cases where gateway feeder links experience outage due to meteorological conditions or gateway failure. The handover typically implies that additional traffic is addressed towards another satellite gateway to handle the capacity reduction. Toward this objective, we propose an architecture framework to support GWD using SDN/NFV-enabled satellite ground segment and we evaluate our solution on a proof of concept experimental testbed based on OpenSAND emulated satellite network. The results show that our proposal can provide reasonably flexibility to handle failover and resiliency.
\end{abstract}

Keywords-Satellite network; Network Function Virtualization; Software-Defined Networking; Satellite gateway virtualization; Combined satellite-terrestrial networks; service orchestration

\section{INTRODUCTION}

The $5 \mathrm{G}$ network is expected to bring new unique service capabilities to end users. It will be designed to support natively heterogeneous network technologies to increase capacity, reliability and availability. In this context, the role that satellite communications can bring in the forthcoming $5 \mathrm{G}$ ecosystem is being overhauled to achieve the expected benefits [1]. Technological advances in the satellite domain such as the use of High Throughput Satellites (HTS) in Geostationary Earth Orbit (GEO) [2], satellite constellation, and laser light satellite communication are changing the way that capacity is being brought to the market, reducing the price per bit and making the satellite technology more attractive for existing and future services. Likewise, a range of disruptive initiatives envisioning the use of non-GEO constellations including a large number of low-cost micro-satellites [3], CubeSat and CanSat complemented with adequate ground infrastructure might come to fruition and will realize their full potential in the forthcoming years, anticipating a further reduction in the cost of transmitting a bit over a satellite link while providing a high reliability, global coverage and very low latency.
In this context, the introduction of emerging technologies such as Software Defined Networking (SDN) and Network Function Virtualization (NFV) within the satellite ground networks is anticipated to be a necessary step in their evolution [4][5]. Both SDN and NFV technologies can bring sustainable flexibility to Satellite Network Operators (SNOs), reducing both operational and capital expenses in deploying and managing SDN/NFV-compatible networking equipment as well as facilitating the integration and operation of combined satellite and terrestrial networks [6]-[8]. At the end of the day, SDN/NFV technologies are expected to better prepare SNOs for tomorrow's softwarised communications networks [9] to be fully integrated in the $5 \mathrm{G}$ era.

Considering the actual growth of raw satellite capacity, by 2020, the satellite will supply at least 1 Tbps capacity. To meet this bandwidth requirement, migration to higher bands of the spectrum is necessary. While higher bands offer more bandwidth, they are also more sensitive to atmospheric perturbation. Today's widely used fading mitigation techniques (FMT) such as waveform adaptation (adaptive coding and modulation, data rate adaptation, etc.) and power control cannot manage successfully these issues. To address problem related to capacity enhancement, fading and failure, it is possible to use multiple interconnected satellite gateways, distributed geographically, and working together to feed the same satellite as illustrated in Fig. 1. These gateways interconnected in a satellite diversity network, can operate in load-sharing (diversity) or in a warm standby with identical configuration to increase resiliency, continuity, and providing higher resource efficiency. In the context of VITAL project, Sat-Cloud-RAN concept has been proposed to virtualize the satellite gateway to run on cloud-based platform as software modules. Multiple instances of virtual satellite gateways are used with possibility of running diversity and redundant gateway either inside one Sat-Cloud-RAN or on multiple geographically distributed SatCloud-RAN environments. This paper describes the support of Satellite Gateway Diversity (GWD) solution in the forward link using SDN/NFV as two enabling technologies to provide next generation satellite system with higher capacity enhancement, failover and resiliency management. Our contribution extends the work presented in [10] where the authors focused on prediction algorithms to orchestrate SDN-based gateway 
handover operations without assessing the management of VNFs.

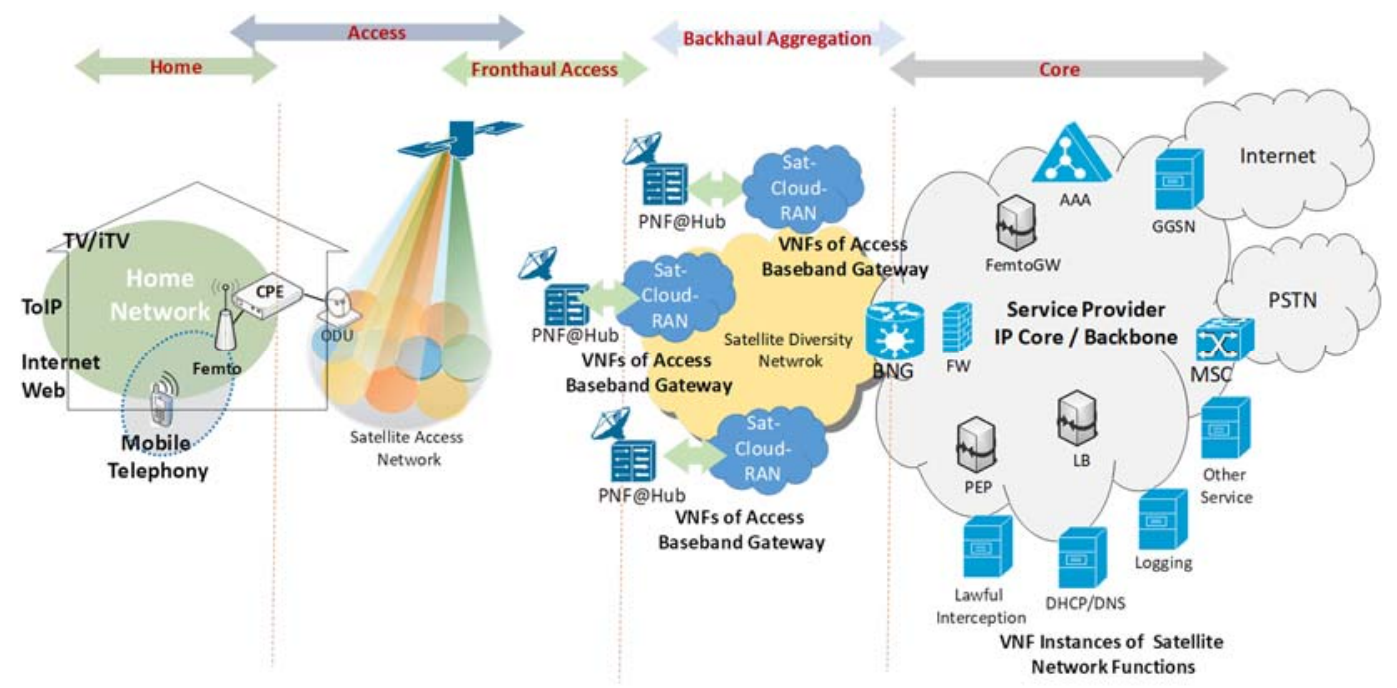

Fig. 1. Target network model for GWD

\section{ARCHITECTURE FRAMEWORK FOR SDN/NFV-ENABLED SATELLITE GROUND SEGMENT SYSTEMS}

The satellite gateway (GW) is the central component in a satellite network. A satellite GW typically comprises: (1) the Out-Door Unit (ODU), composed of the antenna and radio head units; (2) the Satellite Baseband Gateway (SBG) subsystems for satellite access and transmission; and (3) a set of Satellite Network Functions (SNFs), in charge of the L2/L3 interconnection with the satellite core network, Performance Enhancing Proxy (PEP) functions (e.g. TCP optimization, content caching) and other generic functions such as firewalling. The virtualization of the satellite $\mathrm{GW}$ is studied in [11], identifying different variants with regard to which functions can be implemented as Virtualized Network Functions (VNF) and which remain as specialized hardware appliances (i.e. Physical Network Functions, PNFs). Without being conditioned to any particular variant, in the following we denote SNF-VNF, to the implementation of the SNFs as VNFs, SBG-VNF to the implementation of part of the SBG functions as VNFs and SBG-PNF to the non-virtualized part of the SBG functions. The chaining, orchestration and dimensioning of these entities is out-of-the scope of this paper.

\section{A. Physical network infrastructure}

As depicted in Fig. 2, the physical network infrastructure of an SDN/NFV-enabled satellite networks is assumed to consist of the following elements:

- $\quad$ NFV Infrastructure-Point(s) of Presence (NFVI-PoP(s)) for the deployment of SNF-VNFs. The main resources in this NFVI-PoP are network, computing (CPU) and storage.
- NFVI-PoP(s) for SBG-VNFs. This represents the virtualization infrastructure over which the SBG functions would be deployed. This infrastructure is likely to be located in or close to the satellite hub premises.

- One or several SBG-PNFs. These elements host the non-virtualized part of the satellite gateway, which is directly connected to the ODUs.

- Transport network between the several NFVI-PoPs (backhaul) and between the NFVI-PoP where the SBGVNFs are run and the location that hosts SBG-PNFs (fronthaul).

B. Virtualized satellite network

On top of the above described physical network infrastructure, one or several virtualized satellite networks could be deployed, as illustrated in Fig. 2. A Virtualized Satellite Network (VSN) is conceived here as a satellite network in which most of their functions are supplied as software components running in one or several NFVI-PoPs of the SNO physical network infrastructure. Each of the VSNs may include a variety of different entities (e.g. PEP, security). In particular, as illustrated in Fig. 1, the following entities could constitute part of a given VSN:

- One or several SNF-VNFs and one or several SBGVNFs. All these functions are part of the data plane processing.

- Control applications and SDN controllers (all running as VNF instances) for the control and management planes of the VSN.

- $\quad$ Network Management (NM) and Element Management (EM) functions of the VSN. This provides a package of 
functions (to be used by the operator of a particular VSN) for the management of the VSN (e.g. FCAPS management).

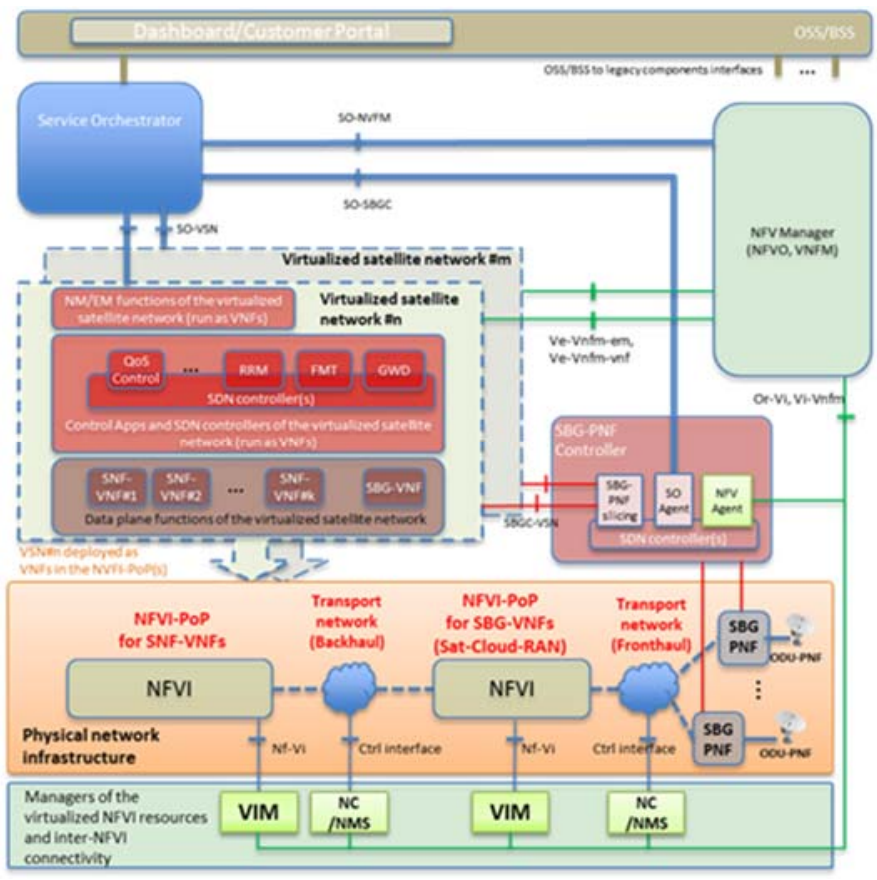

Fig. 2. Architecture reference model of the SDN/NFV-enabled satellite ground segment

\section{Management components}

The creation and management of the lifecycle of the VSNs is realized through a set of functional entities within the SNO domain. In particular, VSNs can be instantiated, terminated, monitored, and modified through the following management entities:

- Service Orchestrator (SO). Decides on the composition and capabilities of the VSN. Once a network service is defined, the SO provides the necessary deployment templates to the NFV management infrastructure. In parallel, the SO allocates and configures the required resources in the SBG-PNFs that will be used by the VSN.

- NFV management entities. Comprised of the NFV Manager, the Virtual Infrastructure Managers (VIMs) for each involved NFVI-PoP and the Network Controllers (NC)/Network Management Systems (NMS) in charge of the connectivity between the NFVIPoPs and between NFVI-PoPs and SBG-PNFs. The NFV Manager handles NFV Orchestration (NFVO) and VNF Management (VNFM) functions as per ETSI ISG MANO specifications.

- SBG-PNF-Controller. This element hosts a set of control programs and a SDN controller to manage the pool of SGB-PNFs. Through a SO agent application, the $\mathrm{SO}$ can request the allocation of SGB-PNFs resources for a given VSN. Therefore, the SBG-PNF-Controller will be in charge of slicing the resources of the SBG-
PNF so that a logically isolated portion of those resources is allocated to a particular VSN. Through a NFV agent, the SBG-PNF-Controller will also provide support to the NFV Manager. In addition, the SBGPNF-Controller provides a SDN abstraction of the allocated resources so that control and management of these resources can be integrated within the VSN.

- SNO's OSS/BSS components such as dashboards/customer portals that the customers of the SNO can use to order the provisioning of VSNs and related SLA management.

\section{SATEllite GATEWAy Diversity SOLUTION FrAMEWORK}

The aim of gateway diversity is to provide a network operator with capability of performing diversity scheme in the forward link by allowing multiple gateways, geographically distributed, to feed simultaneously the satellite to accommodate a high capacity aggregation using a high number of beams. This satellite gateway diversity implies:

- Inter-gateway handover technique to cope with the cases where gateway feeder links experience outage due to meteorological conditions or failure. The handover typically implies that additional traffic is addressed towards another gateway to handle the capacity reduction of the affected gateway.

- Permanent monitoring of hub and radio resources to detect the outage, failure or any problem.

- Reconfiguration of the network capacity, optimizing traffic engineering, routing table and forwarding elements of the core satellite network provider to support temporarily capacity changes.

We consider in the following, the problem of gateway diversity (GWD) to provide failover and resiliency for the traffic passing through the satellite network. We will interest in two cases of GWD providing failover mode as illustrated in Fig. 3.

- GWD within a particular NFVI-PoP that hosts SATCloud-RAN resources in terms of pool of active and passive SBG-VNF. In this case, the GWD is done in intra Sat-Cloud-RAN. As illustrated in Fig. 3. (a), if one active SBG-VNF fails, the passive SBG-VNF will take over seamlessly.

- GWD for geographically distributed NFVI-PoPs used to balance the traffic to other region to continue to serve the same remotes. In this case, the GWD is done in inter Sat-Cloud-RANs (see Fig. 3. (b)).

Furthermore, in the context of NFV architecture, GWD solution benefits from the possibility to instantiate dynamically some VNFs either to deal with extra charges needed or to replace a VNF such as in case of failure. In case of SBG-VNF failure, it is possible to instantiate a new working SBG-VNF either on the same NFVI-PoP or inside other NFVI-PoPs. Once instantiated, the traffic must be seamlessly directed to this new instance. In all these cases, the network service composition must be updated to take the current working VNF. 

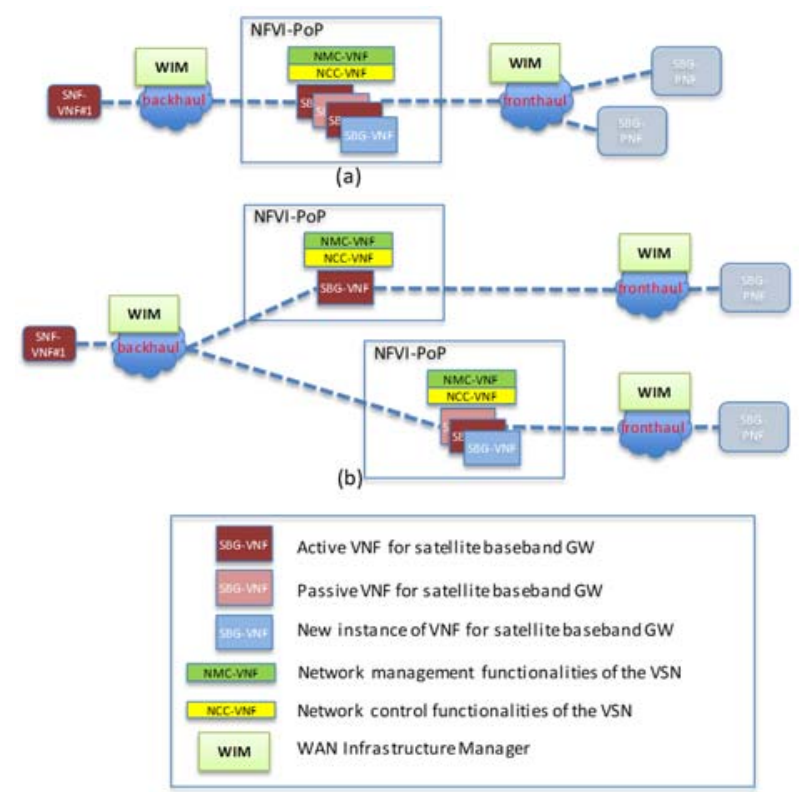

Fig. 3. Architectural view of a VSN with SGW support

\section{A. Intra Sat-Cloud-RAN Failover Diversity}

In this mode, we suppose a VSN composed of a pool of active and passive SBG-VNF hosted in the same NFVI-PoP forming the SAT-Cloud-RAN. The pool of passive SBG-VNFs will be used when any active SBG-VNFs fails. Toward this end, a network monitoring entity is used to continuously monitor the current SBG-VNFs state and to determine any failure or operation condition (e.g. misbehaviour, fading situation that cannot be properly addressed only with the adaptive modulation and coding functions of the SBG-VNF) from the active SBGVNF that necessitates to balance the traffic to the passive SBGVNF using traffic steering activation from the SDN controller. The following procedure (see Fig. 4) illustrates the scenario for Intra Sat-Cloud-RAN failover diversity. The transfer of context from active to passive SBG-VNFs can be quickly performed on the same Sat-Cloud-RAN. This context contains the configuration parameters actually used by the active SBG-VNF in terms of control and management information that is gathered at the levels of NMC-VNF and NCC-VNF. The passive SBG-VNF can then inherit from the context of the active SBG-VNF locally. This GWD scheme allows the output Base Band Frames (i.e. BBFrames) from the SBG-VNFs gateway to be seamlessly directed to the SBG-PNFs with minimum interruption delay. Fig. 4 describes the process in different steps that reflect how the failure of SBG-VNF_1 is handled within the VSN. Indeed, before SBG-VNF_1 failure, the traffic flows from SNF-VNF to SBG-VNF_1 to the SBGPNF. Once the failure is detected and corrected, the traffic will flow now from SNF-VNF to SBG-VNF_2 to the SBG-PNF. The following steps illustrate how this failover if managed by the GWD procedure:

Step 1: The NMC-VNF continuously monitor both active and passive SGB-VNFs (SBG-VNF_1 and SBG-VNF_2 in this example) to retrieve their state and health conditions. For example, a simple monitoring can be done using ICMP Echo request, which needs in response an Echo reply.

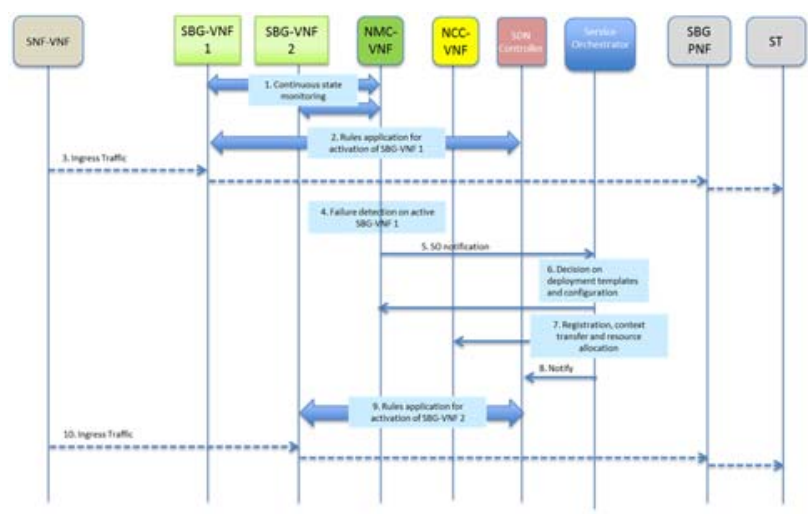

Fig. 4. Illustration of Intra Sat-Cloud-RAN Failover Diversity

Step 2: We suppose in normal network operation that the SBGVNF_1 is used by default. The SDN controller with appropriate routes and configuration parameters configures the rules used in the composition of the network service basically.

Step 3: The WAN ingress traffic flows now from SNF-VFN to SBG-VNF_1 and then to SBF-PNF.

Step 4: Failure is detected on the current active SBG-VNF and reported to the service orchestrator (SO) in step 5.

Step 6: SO takes a decision on new configuration that will take into account the passive SBG-VFN_2 that will be used in replacement of SBG-VNF_1. If needed a new instance of the SBG-VNF can be instantiated.

Step 7: The SO request the context transfer on both NMC-VFN and NCC-VNF.

Step 8: The SDN controller is notified that the SBG-VNF_2 is ready to handle the traffic.

Step 9: SDN controller pushes new rules for the activation of SBG-VNF_2.

Step 10: Traffic can now flow from SNF-VFN to SBG-VNF_2 and then to SBF-PNF.

\section{B. Inter Sat-Cloud-RAN Failover Diversity}

This mode is similar to the intra Sat-Cloud-RAN failover diversity, however the switching occurs on different NFVIPoPs that may be geographically separate.

C. Intra or Inter Sat-Cloud-RAN Failover Diversity with onfly SBG-VFN instantiation

This mode implies that a new working SBG-VNF has be instantiated on the fly to overcome any failure on the active SBG-VNFs. This instantiation can be done locally on the same Sat-Cloud-RAN or on geographically separated Sat-CloudRAN.

\section{Proof of Concept And EXPerimental AsSESSMENT}

We consider a target network architecture composed M [1..M] Sat-Cloud-RAN environments. Each Sat-Cloud-RAN environment $\mathbf{i}$ is composed of a pool of $\mathbf{N i}$ active gateways with Pi redundant $(\mathbf{N i}+\mathbf{P i})$. We expose each active gateway to specific parameters like fading, up time and failure. 


\section{A. Architecture of a Sat-Cloud-RAN test platform}

We implement a Sat-Cloud-RAN test platform as a fully virtualized environment that uses KVM for virtual machine virtualization (hypervisor) and Open vSwitch (OvS) for network virtualization (software defined networking, SDN) interconnecting the different components of the emulated testbed. Fig. 5 illustrates the components implemented to demonstrate the GWD.

Each Sat-Cloud-RAN being a separated set of virtual machines (VMs) which are used to run the different components of OpenSAND platform [12]. One platform is composed of at least a satellite manager, a satellite payload, a satellite terminal, one primary gateway, and one secondary gateway $(1+1$ redundancy schema). We also provide an additional VIM equipped with a Ryu SDN controller in charge of performing the appropriate logic for traffic steering between the primary and the secondary gateways inside the SAT-Cloud-RAN test platform. The design of this platform has allowed a lightweight environment capable of short deployment time and almost instant instantiation of the OpenSAND components.

The idea of GWD (gateway diversity) is to enable a warm standby using identical configuration of satellite gateway inside the Sat-Cloud-RAN platform. Toward this objective, we developed a Ryu application as a SDN controller to decide how the traffic is being managed inside the platform. Furthermore, we constantly monitor the satellite gateways liveliness in order to detect any failure or loss of capacity and then to decide to steer the traffic to the appropriate functional gateway.

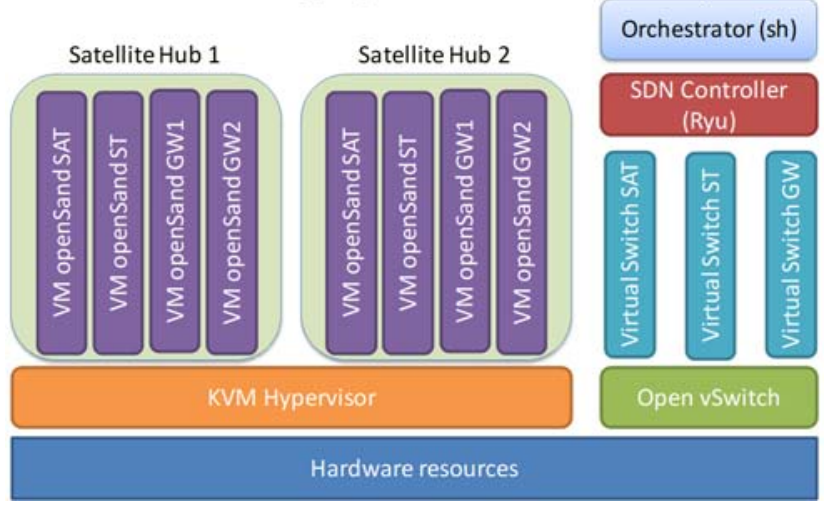

Fig. 5. Implementation of gateway diversity solution

\section{B. Description of the test scenario}

The gateway diversity scheme inside one Sat-Cloud-RAN is able to provide reliable data transmission in case of fading (due to meteorological events) or interruption. In this case, the traffic can be steered to others gateways connected to the satellite diversity network.

Our test scenario aims to demonstrate how the gateway failover is managed using both NFV and SDN paradigm. While NFV allows to instantiate a new operational gateway on the fly, SDN capabilities enables to control the network centrally, in flexible manner by enabling a smart traffic steering. To achieve this objective, the implemented platform provides a logic of failover capability inside Ryu controller which allows to automatically steer the traffic to another operational satellite gateway (that can be instantiated on the fly or one already instantiated) if the primary one can no longer be used.

Technically, the traffic intended to one gateway can be forwarded to another gateway using traffic controller over the OvS bridge port number. In fact, since our platform implements an Open vSwitch solution, all the VMs are connected to an OvS bridge. This latter assigns a port number to each of them. This port number will be used thereafter to forward the L2 traffic to a specific VM regardless of the MAC addresses (Open vSwitch handles this part).

At Layer L3, a default IP gateway has to be defined in the routing policy database (with route command) of the ingress node of our platform. Since it cannot be any of the satellite gateways (because one of them could eventually fail and no longer be alive), we implement a virtual IP router capable of handling the IP packet routing to the appropriate gateway. Meaning that all the traffic intended for this virtual IP will be handled by our Ryu application and will be steered to the right satellite gateway. This is a reliable solution since the virtual IP does not depend on the actual IP configuration of the satellite gateways at all.

We configure the OvS to notify the SDN controller to retrieve the forwarding rule on each received packet need to be handled. We implement our forwarding rules to focus mainly on the traffic intended toward the virtual IP address, which is in fact, the traffic intended for one of the satellite gateway. Based on this rule, the SDN controller informs the OvS how to handle a packet by forwarding the L2 frame to the selected satellite gateway using an OpenFlow FlowMod. This FlowMod is a simple OpenFlow rule telling the OvS to automatically forward the incoming traffic intended for the virtual IP, directly to the chosen satellite gateway.

To optimize the overall behavior, we introduce a timeout delay allowing using the same rule until the expiration of a timer or until the SDN controller sends again a new rule, which replaces the previous one. The purpose of this scheme is to increase the network performance in terms of reducing the latency needed of the SDN controller when asking for the rule for each packet. This rule is obviously going to expire after some time to make the switch asking the SDN controller after a while, whether it should pursue the steering to the same satellite gateway or to steer it to another.

\section{Description of the obtained results}

Our test scenario consists in simulating a failure of the primary satellite gateway (called GW1) and switching all the traffic toward the secondary satellite gateway (called GW2) inside the OpenSAND emulator. For the first test scenario, we prepare in advance the GW2, which is in standby mode waiting for any traffic. It is worth noting that GW1 and GW2 are being configured with the same parameters and having the same configuration so that the step of transferring the configuration context is facilitated. Once the failure is detected, the incoming traffic is automatically switched to the right gateway using the developed Ryu application. Fig. 6 shows the OpenSAND configuration as described by OpenSAND manager.

The carrier setting for forward band configuration, in our test, was 40 Mbauds. The MODCOD configuration was set in ACM 
with modulation available from QPSK $1 / 4$ to 32 APSK 9/10. The coding is then negotiated at the simulation start.

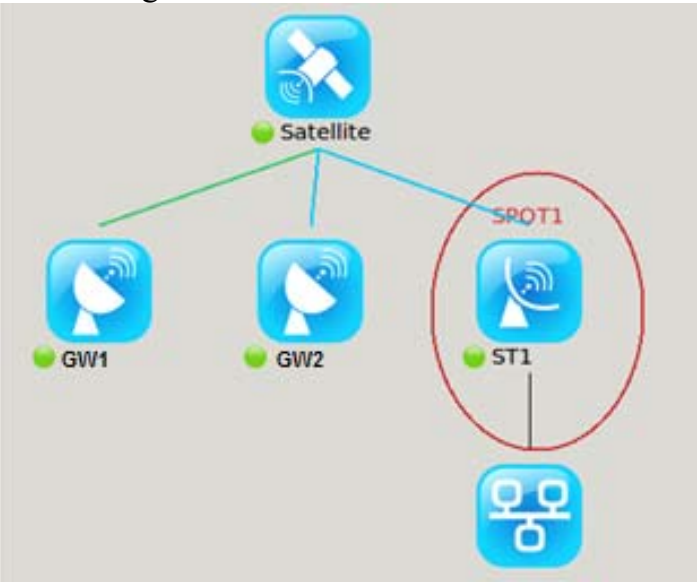

Fig. 6. Snapshot of the OpenSAND emulation as described by OpenSAND Manager.

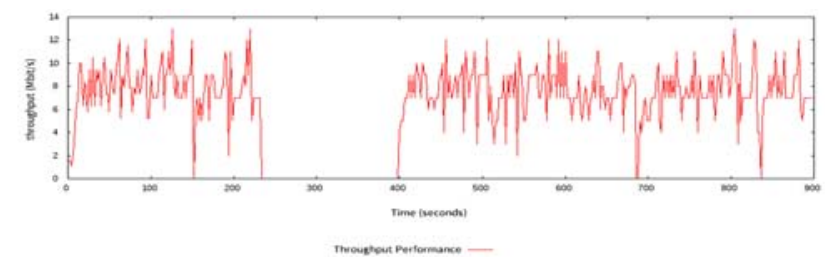

Fig. 7. Obtained results for traffic received from GW1 (time from 0 s to $240 \mathrm{~s}$ ) and from GW2 (time from 400s - 900s).

To evaluate the GWD, we configured a traffic sender and receiver passing through the Sat-Cloud-RAN platform. During the first seconds (230s), the TCP traffic goes normally through the primary gateway (GW1). We manually turn-off GW1 at time $\mathrm{t}=240 \mathrm{~s}$ to simulate a brutal failure that has occurred in the gateway, leading to a long downtime. The SDN controller was notified about this failure and has installed a new rule (OpenFlow FlowMod) informing the OvS how to forward properly the traffic toward the secondary gateway (GW2) as illustrated in Fig. 7. The elapsed time between time $t=240$ s and $\mathrm{t}=400 \mathrm{~s}$ was needed to restart the OpenSAND emulation to allow seamless traffic steering over GW2. During all our test we were be able to steer the traffic automatically, and to demonstrate how to implement GWD by relaying on both SDN and NFV technologies. However, our platform may still need some improvement to allow automatic instantiation of gateway and to restart the OpenSAND simulation process promptly. We are actually implementing a solution for multiple geographically distributed Sat-Cloud-RAN environments.

\section{CONCLUSIONS}

The adoption of SDN and NFV technologies into the satellite domain is seen as a key facilitator to enhance the delivery of satellite communications services and achieve a better integration of the satellite component within the $5 \mathrm{G}$ ecosystem. This paper has described a gateway diversity (GWD) mechanism proposed on top of an architecture framework that uses SDN/NFV-enabled satellite ground segment system and that supports on-demand dynamic orchestration of satellite communications services. This study is an experimental assessment as a proof on concept aiming to provide failover and resiliency for the traffic passing through the satellite network. It was illustrated how an SDN-based application and VNF on demand instantiation can be used to facilitate a complex traffic engineering solution. In particular, we show traffic steering mechanism in the satellite network to allow the satellite operator to conduct its traffic to specific region / domain / gateway and this as needed. The assessment shows a use-case of a traffic that is forwarded to a secondary satellite gateway in case of failure of primary satellite gateway. Given this, the satellite operator will have the ability to design a more complicated GWD solution.

\section{ACKNOWLEDGMENT}

Research leading to these results has received funding from the European Union's H2020 Research and Innovation Programme (H2020-ICT-2014-1) under the Grant Agreement H2020-ICT-644843.

\section{REFERENCES}

[1] M. Corici, A. Kapovits, S. Covaci, A. Geurtz, ID. Gheorghe-Pop, B. Riemer, A. Weber. "Assessing satellite-terrestrial integration opportunities in the 5G environment", September 2016

[2] Fenech, H.; Amos, S.; Tomatis, A.; Soumpholphakdy, V., "High throughput satellite systems: An analytical approach," IEEE Transactions on in Aerospace and Electronic Systems, January 2015

[3] ARTES programme, "ESA announces dedicated support for the development of megaconstellations", Last updated July 2015.

[4] H2020 VITAL research project website at http://www.ict-vital.eu/

[5] Bertaux L., Medjiah S., Berthou P., Abdellatif S., Hakiri A., Gelard P., "Software Defined Networking and Virtualization for Broadband Satellite Networks". IEEE Communications Magazine, March 2015

[6] Sacchi, C.; Bhasin, K.; Kadowaki, N.; Vong, F., "Toward the "space 2.0" Era [Guest Editorial]," Communications Magazine, IEEE , vol.53, no.3, pp.16,17, March 2015

[7] NetWorld2020's - SatCom WG The role of satellites in 5G, Version 5 31th July 2014

[8] 3GPP TR 22.891 V1.1.0, "Feasibility Study on New Services and Markets Technology Enablers; Stage 1 (Release 14)", November 2015

[9] R. Ferrus, H. Koumaras, O. Sallent, G. Agapiou, T. Rasheed, M.-A. Kourtis, C. Boustie, P. Gelard, T. Ahmed, SDN/NFV-enabled satellite communications networks: Opportunities, scenarios and challenges, Physical Communication, November 2015

[10] M. Mongelli, T. De Cola, M. Cello, M. Marchese and F. Davoli, "Feederlink outage prediction algorithms for SDN-based high-throughput satellite systems," 2016 IEEE International Conference on Communications (ICC), Kuala Lumpur, 2016, pp. 1-6.

[11] T. Ahmed, E. Dubois, J.-B Dupé, R. Ferrús, P. Gélard, and N. Kuhn, Software-defined satellite cloud RAN. Int. J. Satell. Commun. Network, 2017.

[12] The OpenSAND platform avaiable at : http://opensand.org/ 